\title{
Stereotactic body radiotherapy for lung metastases in a patient with giant cell tumor of bone: a case report and literature review
}

\author{
Liwen Feng ${ }^{\sharp}$, Ting Ye ${ }^{\#}$, Jieying Zhang, Siyue Yuan, Yuting Chen, Jing Chen \\ Cancer Center, Union Hospital, Tongji Medical College, Huazhong University of Science and Technology, Wuhan, China \\ \#These authors contributed equally to this work. \\ Correspondence to: Jing Chen. Cancer Center, Union Hospital, Tongji Medical College, Huazhong University of Science and Technology, Wuhan \\ 430022, China. Email: chenjingwh@hust.edu.cn.
}

\begin{abstract}
Giant cell tumor of bone (GCTB) is a rare borderline tumor which can develop lung metastasis. Guidelines for patients with multiple pulmonary metastases recommend systemic therapies, such as denosumab and interferons (IFNs). However, when both of these treatment approaches fail, no satisfactory options are available. Thus, additional treatments for GCTB after failure of standard treatment would be beneficial. Here we present a patient with GCTB and multiple pulmonary metastases who was treated with stereotactic body radiotherapy (SBRT) after failure of surgery, denosumab, and bisphosphonates (BPs). This is the first well-documented report of a patient with metastatic GCTB who received lung SBRT after the failure of systemic therapy and achieved a favorable response. Some of the patient's pulmonary metastases were treated using SBRT (44 Gy/4 F). The long diameters of the irradiated nodules decreased 58.2\% from baseline in the 30 months after SBRT. Moreover, the peritumoral volume of another nodule also shrank by $29.1 \%$ after receiving a low-scatter dose of $7.6 \mathrm{~Gy} / 4 \mathrm{~F}$, which was too small to have induced tumor regression. No obvious adverse events were observed during SBRT or in the follow-up period. Our case provides clinical evidence that SBRT may be a safe and effective method to treat metastatic GCTB and can produce a low-dose radiation-induced abscopal response, suggesting that immune responses can contribute to GCTB regression. In addition, we reviewed publications regarding treatment recommendations, the prospects for SBRT application, and possible effects of abscopal responses on GCTB.
\end{abstract}

Keywords: Giant cell tumor of bone (GCTB); lung metastasis; stereotactic body radiotherapy (SBRT); abscopal effect; case report

Submitted Nov 18, 2021. Accepted for publication Jan 27, 2022.

doi: $10.21037 / \mathrm{atm}-21-6575$

View this article at: https://dx.doi.org/10.21037/atm-21-6575

\section{Introduction}

Giant cell tumor of bone (GCTB) is a rare borderline tumor that presents as a locally aggressive lesion. GCTB comprises $3-5 \%$ of primary bone tumors in Western countries and $13.7-17.3 \%$ in Asian countries $(1,2)$. GCTB lesions are unpredictable, with some progressively destructive and prone to local recurrence. Almost $4 \%$ of GCTBs develop hematogenous metastases, usually to the lung, and are associated with inconsistent prognosis. The majority of studies have reported that this type of benign lung metastasis progresses slowly and that patients exhibit relatively long survival (3), although there is some diseaserelated mortality in this group. Siebenrock et al. followed up 23 patients with GCTB and pulmonary metastases and found that 4 of them died due to disease progression, corresponding to a mortality rate of $17.4 \%$ (4).

Surgery remains the main treatment modality for GCTB. In addition, the National Comprehensive Cancer Network guidelines recommend systematic treatment such as denosumab or interferon (IFN) for patients with

\footnotetext{
$\wedge$ ORCID: 0000-0002-7279-7050.
} 
metastases that are not amenable to resection. However, effective treatments for patients in which these approaches fail are currently lacking (5). Further, due to the long survival of patients with GCTB, treatment-induced side effects warrant particularly close attention. Although there have been studies reporting novel treatments for metastatic GCTB involving various antiangiogenic drugs and checkpoint inhibitors, they have been limited to case reports or have resulted in drug toxicity (6-8). Herein, we present the case of a patient with GCTB and multiple pulmonary metastases who received stereotactic body radiotherapy (SBRT) following failure of standard systemic treatment and achieved a good response without obvious adverse events. To our knowledge, this is the first report of a patient with metastatic GCTB who received lung SBRT after systemic therapy failure. The duration of the response to SBRT in this patient has exceeded even that of denosumab, which is currently recognized as the most effective systemic therapy. Interestingly, a low-dose radiation-induced abscopal response was also observed in this case, suggesting a potentially important role for radiotherapy in GCTB.

We present the following article in accordance with the CARE reporting checklist (available at https://atm. amegroups.com/article/view/10.21037/atm-21-6575/rc).

\section{Case presentation}

A 29-year-old female attended hospital in February 2010 complaining of persistent lumbosacral pain. She had no medical history of malignancy or related family history. Pelvic computed tomography (CT) revealed an expansive sacral mass. In March 2010, resection of the sacral mass was performed at another institution and GCTB was diagnosed by histopathological examination. Five years later, multiple metastases were detected in her lungs during routine examination and confirmed by biopsy. She was treated with denosumab $(120 \mathrm{mg})$ every 4 weeks (Q4W) until progression occurred 26 months later. Then, bisphosphonates (BPs) (4 mg, Q4W) were administered intravenously. However, 10 months later, the pulmonary metastases progressed again.

After the failure of BPs, the patient was admitted to our hospital in June 2018. She presented in good general condition [Eastern Cooperative Oncology Group (ECOG) performance status, 0] with some perineal paresthesia and obvious respiratory sounds on physical examination. Contrast-enhanced CT scan of the chest and pelvis was performed to evaluate the lesions. Solid and enhanced lesions with irregular contours were observed in both lungs, while no definite recurrence was detected in the pelvis. Four of the lung tumors were measurable and these were located in the anterior basal segment of left lower lobe, the outer basal segment of right lower lobe, the posterior basal segment of right lower lobe, and the anterior basal segment of right lower lobe, with long diameters of 20.44, 20.83, 16.99, and $13.85 \mathrm{~mm}$, respectively (Figure 1). We numbered the lung nodules 1, 2, 3, and 4 in sequence, as shown in Figure 1.

The patient refused any further systemic medication because of the previous unsatisfactory response to therapy. Given the sizes of the pulmonary lesions $(<50 \mathrm{~mm})$, we conducted SBRT on 3 of them (nodules 1, 2, and 3) on June 11, 2018. The prescribed dose was $44 \mathrm{~Gy} / 4 \mathrm{~F}$, and a $72 \%$ isodose line encapsulated the edge of the lesions (Figure 2). SBRT was not administered to nodule 4 because it was close to the top of the diaphragm and the accuracy of radiotherapy would have been significantly affected by respiratory movements. Nevertheless, nodule 4 received a low scatter dose of $7.6 \mathrm{~Gy} / 4 \mathrm{~F}$ in total (Figure 2). The patient exhibited good adherence and tolerability during radiotherapy.

After SBRT, the patient was followed up and tumors assessed according to Response Evaluation Criteria in Solid Tumours (RECIST) 1.1. The size of the irradiated nodules increased approximately $4.1 \%$ at the end of the first month but decreased by almost $58.2 \%$ from baseline by the end of the $30^{\text {th }}$ month after radiotherapy. Interestingly, an unanticipated low-dose radiation-induced abscopal response was observed. The size of nodule 4 , which was not included in the planning target volume (PTV), shrank continuously and reduced $29.1 \%$ compared with baseline at the end of the $30^{\text {th }}$ month. Grade I radiation-associated pneumonia [National Cancer Institute Common Terminology for Adverse Events (NCI-CTCAE), version 4.0] was observed during follow-up. A clinical timeline is presented in Figure 3.

All procedures performed in this study were in accordance with the ethical standards of the institutional and/or national research committee(s) and with the Helsinki Declaration (as revised in 2013). Written informed consent was obtained from the patient for publication of this case report and accompanying images. A copy of the written consent is available for review by the editorial office of this journal.

\section{Discussion}

As a borderline tumor, GCTB is both locally destructive 

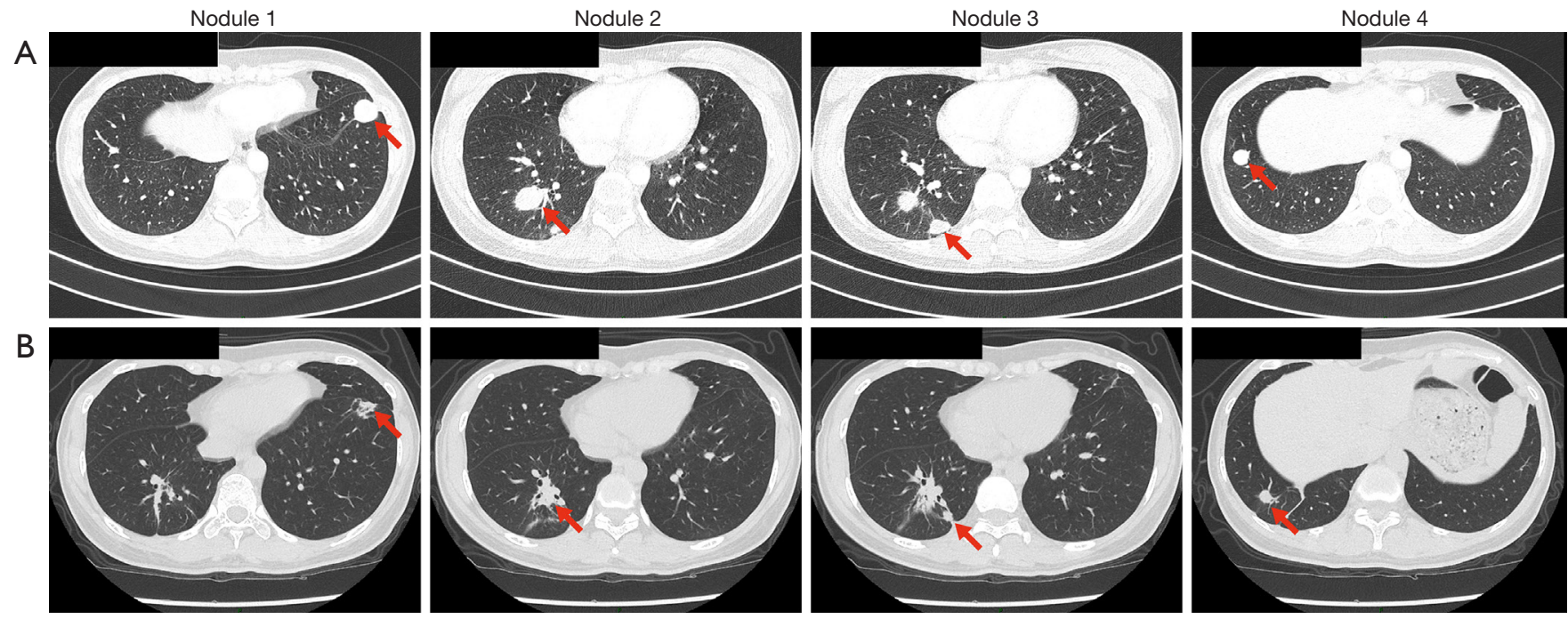

Figure 1 CT images of the lung metastases before (A) and 30 months after SBRT (B). The red arrows point to the lesions. CT, computed tomography; SBRT, stereotactic body radiotherapy.
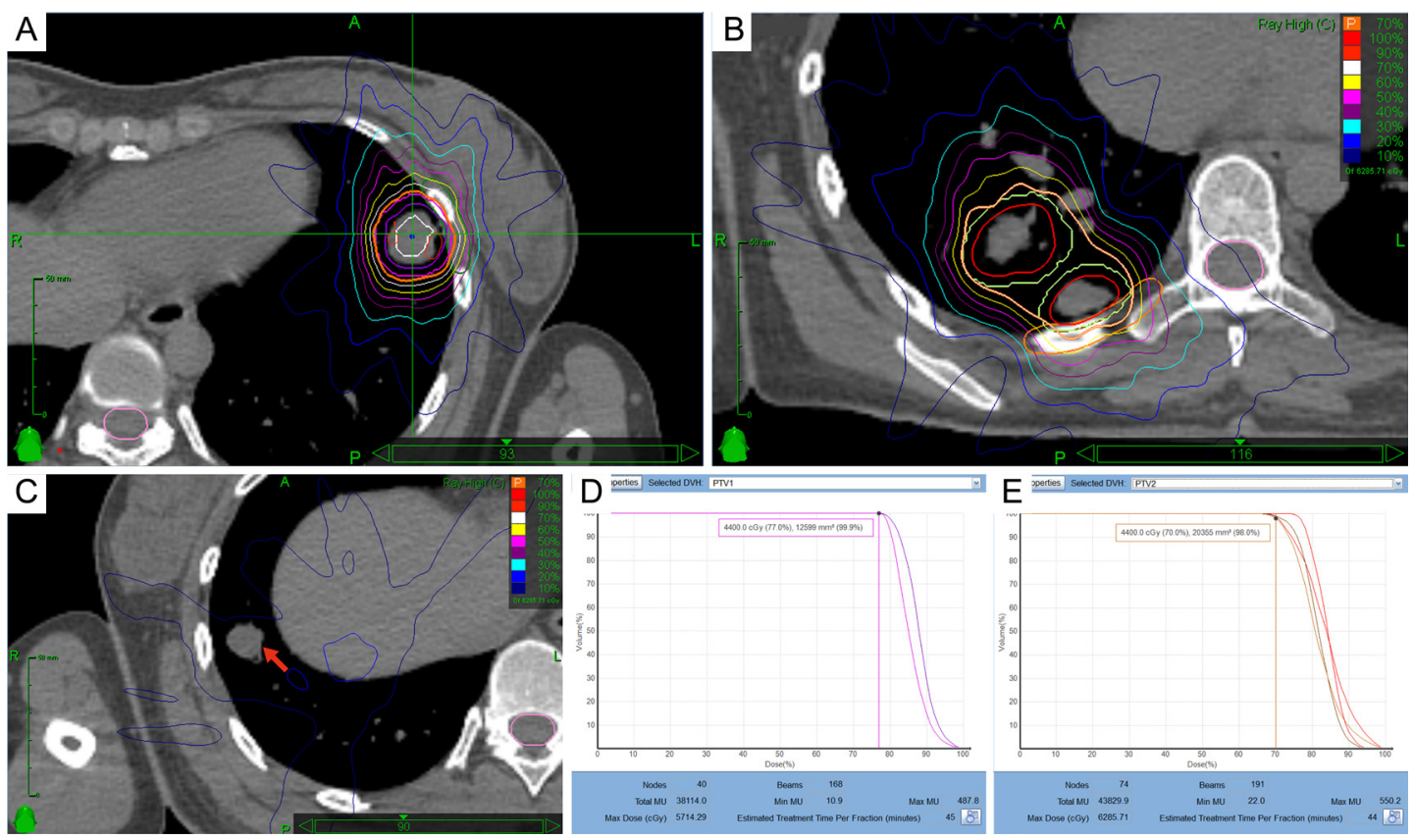

Figure 2 Dose distributions for nodule 1 (A), nodule 2 and 3 (B), while nodule 4 is around the edge of $10 \%$ isodose line (C). DVH for nodule 1 (D), nodule 2 and 3 (E). The red arrows point to the lesions. DVH, dose-volume histogram; PTV, planning target volume; MU, monitor unit.

and can generate benign lung metastases. Surgery is considered the gold-standard treatment for localized lesions and resectable metastases; however, for a few patients with unresectable lesions, the treatment options are limited. At present, systemic therapy is recommended in surgically unsalvageable GCTB, with denosumab the most common choice. Denosumab, an antibody against receptor activator of nuclear factor- $\kappa \mathrm{B}$ ligand (RANKL), was developed in 


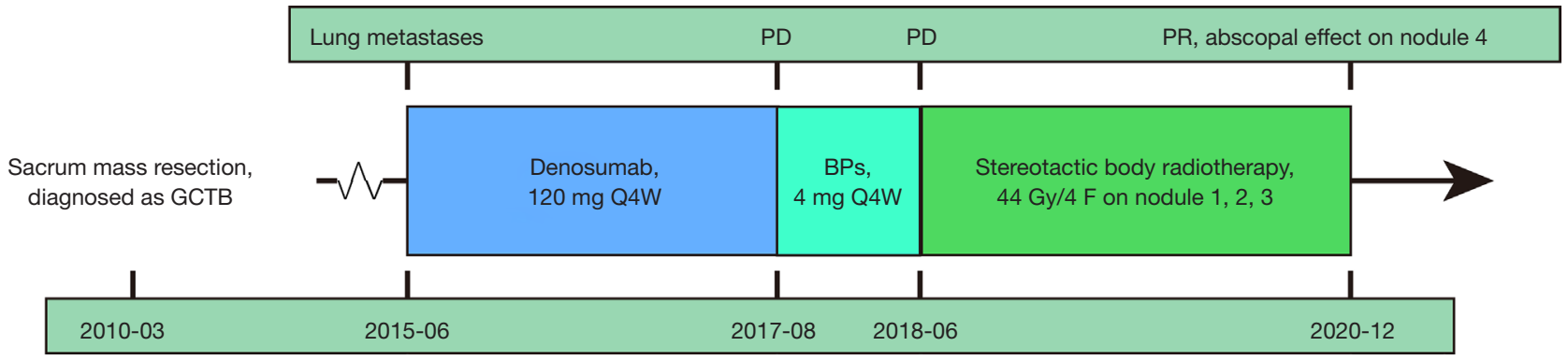

Figure 3 Timeline of the clinical course. PD, progressive disease; PR, partial response; GCTB, giant cell tumor of bone; Q4W, every 4 weeks; BPs, bisphosphonates.

response to recognition of the role played by RANKL in GCTB $(9,10)$. Denosumab was approved by the US Food and Drug Agency in 2013 for the treatment of adults and skeletally mature adolescents with unresectable GCTB, and in cases where resection may result in severe morbidity. A phase II study in 2010 provided the foundation for this approval (10). The primary endpoint was tumor response, defined as elimination of at least $90 \%$ of giant cells or no radiological progression of the target lesion up to week 25 , and $86 \%$ (95\% confidence interval, $70-95 \%$ ) of patients given denosumab exhibited tumor response. Our patient was first treated with denosumab following diagnosis of multiple lung metastases and the response duration was 26 months. According to the 2021 National Comprehensive Cancer Network guidelines, IFN is a secondary recommendation. IFN is primarily classified into $\alpha, \beta$, and $\gamma$ subtypes based on antigenic type and has an antitumor role through its antiangiogenic, antiproliferative, and immunomodulatory properties (11). IFNs are also involved in bone metabolism and homeostasis by inhibiting the proliferation and differentiation of osteoclasts (12). However, to our knowledge, the clinical evidence for treatment of GCTB with IFN remains limited to studies of case series, which had variable response rates depending on dosage and duration $(13,14)$, thereby limiting the potential use of IFN in GCTB due to the lack of a standard dose. Further, for such benign metastasis, treatment-related side effects must also be considered.

Prior to being admitted to our hospital, our patient had received BPs at another institution as secondline treatment with a time to second objective disease progression of 10 months. BPs have an inhibitory effect on GCTB and significantly reduce pain, increase mineralization (particularly at the lesion periphery), and increase the mean apoptotic index (15). However, whether
BPs can also control extraskeletal metastasis remains to be determined. The unique structure of BPs, which comprise 2 phosphonate groups attached to 1 carbon atom and 2 lateral chains, forms a 'clasp' that binds almost exclusively to skeletal tissues. Hence, the deposit of BPs in extraosseous tissues such as lung for treatment of extraskeletal metastasis may not be feasible. To date, no clinical data on the use of BPs for the treatment of GCTB extraosseous metastases are available, and further exploration of this approach is required.

Radiotherapy has been used to treat GCTB for decades. However, radiation-induced long-term complications, such as bone deformation and even malignant transformation, have limited its application. With advances in megavoltage equipment, it has been reported that high doses offer safe and effective local control (16). To reduce treatmentinduced long-term complications, radiotherapy with shorter course, hypofractionation, and more precise features has been attracting increasing attention. SBRT allows delivery of a steep dose gradient that can destroy a target volume while minimizing damage to the adjacent normal structures. In our case, the irradiated lesions shrank $58.2 \%$ from baseline after SBRT, and the time to response following radiation was 4 months. This result is similar to those reported from previous studies. Ruka et al. assessed 77 patients with GCTB and unresectable lesions who received megavoltage radiotherapy, with a median radiation dose of 56 Gy (26-89 Gy), a median follow-up of 58 months, and median time to radiological response of 4 months (216 months) (16).

The application of SBRT in GCTB remains controversial, with evidence limited to a few case reports (17-22). The authors of these reports concluded that SBRT offers a radiological regression benefit and improved symptoms. During the follow-up period, none of the 
patients experienced serious adverse effects; however, almost all radiotherapy targets were primary bone lesions, with only 1 case with targeted lung metastases (19). In this study, we provided the first report of a case of metastatic GCTB treated with SBRT after failure of systemic therapy. Surprisingly, this approach achieved a longer response duration than denosumab (at least 30 vs. 26 months). By December 2020, we had followed this patient for 30 months. Tumor response was evaluated as partial response (PR), with additional observation of a low-dose radiation-induced abscopal response.

Nodule 4 was outside of the PTV site but responded to SBRT. The term 'abscopal effect' describes additional regression of the tumor burden in nonirradiated sites after local radiation therapy and was originally defined by Mole in 1953. The emergence of abscopal effects is thought to be related to immune activation (23-24). In the context of sufficient radiation, tumor cell death occurs and intracellular substances are released. This process transfers antigens from the tumor cell to antigen presenting cells (APCs), which activate $\mathrm{CD} 8^{+}$cytotoxic $\mathrm{T}$ lymphocytes (CTLs). These actions culminate in conversion of the irradiated tumor into an in situ vaccine that elicits tumor-specific $\mathrm{T}$ cell activity. Further, the host develops an immune memory, which acts as a powerful weapon against synchronous nonirradiated tumor lesions (23). However, these immunostimulatory effects may be dampened by stronger immunosuppressive effects at distant sites in the absence of sufficient irradiation $(23,24)$. Myeloid cells, hypoxia, immunosuppressive cytokines, and immune checkpoint inhibitor (ICI) ligands, including programmed deathligand 1 (PD-L1), may contribute to an immunosuppressive tumor microenvironment (TME) (24) as they mediate $\mathrm{T}$ cell exhaustion and are associated with the limitations of abscopal responses. Abscopal effects are uncommon in all types of tumors. A systematic review summarized all clinical case reports of abscopal effects from 1969 to 2014 and recorded only 46 cases, despite millions of patients being treated worldwide (25). However, some of the abscopal effect sites included in the review appeared to be close to the irradiated lesions and received low-dose (approximately $10 \%)$ scatter radiation, which likely promoted the abscopal effects.

At present, a common problem is determining how to encourage strong abscopal effects, and several studies have focused on low-dose radiation strategies for distant lesions $(26,27)$. Despite its very poor tumor-killing effect, lowdose radiation is conducive to $\mathrm{T}$ cell recruitment (26). The optimal radiation strategies to facilitate abscopal effects remain to be verified. Larger doses per fraction in situ appear to be associated with the release of more tumor antigens. In addition, to date, low-dose radiation at distant sites is thought to overcome the inhibitory effects of the TME and enhance abscopal responses $(27,28)$. In 2019, Menon et al. analyzed 83 lesions in 26 patients treated with high-dose SBRT (38 receiving low-dose and 45 receiving no-dose) plus ICIs and found that low-dose radiation at distant sites may have increased systemic response rates and boost abscopal effects (27). Klug et al. revealed that lowdose radiation (2 Gy/1 F) can shift the TME toward an M1/ Th1 phenotype, leading to an enhanced T-cell response (29). In a 2020 study by Yin $e t$ al., enhanced abscopal effects were associated with the increased infiltration of $\mathrm{T}$ cells and dendritic cells, which was induced by low-dose radiation in remote secondary tumors (26). Therefore, it is assumed that high-dose radiation, such as SBRT, kills primary tumor cells directly, which increases the release of tumor antigens and assists the generation of an in situ vaccine, whereas low-dose radiation modulates the TME, thereby promoting immunecell infiltration (26-28). Hence, high-dose and low-dose radiation may function synergistically to elicit antitumor immunity and boost abscopal effects. Our case received $44 \mathrm{~Gy} / 4 \mathrm{~F}$ to 3 nodules, while the other nodule received low-dose scatter radiation of $7.6 \mathrm{~Gy} / 4 \mathrm{~F}$ in total, which is within the dose range (5-10 Gy) that, according to current clinical data, provides the best effect $(27,28)$.

Data regarding abscopal effects in GCTB are very rare. To our knowledge, only 1 case has been reported (30). In that case, $30 \mathrm{~Gy} / 10 \mathrm{~F}$ radiotherapy was prescribed for treatment of right pulmonary metastasis. Fluorodeoxyglucose-positron emission tomography CT showed volumetric and metabolic response of a left-side low-dose irradiated pulmonary metastasis 3 weeks later.

There are several other local treatments for GCTB, including radiofrequency ablation (RFA), a prototypical thermal ablation technique. Although to our knowledge, it has not been reported in metastatic GCTB, the application of RFA in GCTB mainly focuses on tumor curettage followed by microwave ablation inactivation of the residual cavity to further reduce the recurrence rate (31). The cytotoxic mechanism of RFA involves dehydration, protein denaturation, and melting of lipid bilayers, due to rapid induction of high intracellular temperature, which leads to immediate coagulate necrosis of tumor cells; however, due to the effects of vascular necrosis in the ablation site and vessel thrombosis in surrounding tissue, immune infiltration 
following RFA is usually poor, unlike SBRT. In recent years, SBRT treatment progress has been reflected by improved tumor responses to immunotherapy $(27,28)$; nevertheless, determining the ideal combined SBRT and ICI treatment regimen remains a challenge, particularly in terms of intervening time and sequence. Prospective comparative studies should be performed prior to resection and in patients with unresectable lesions to explore the optimal timing for intervention of SBRT and ICI. Furthermore, it is also necessary to compare adverse event rates between SBRT plus ICI combination group and single treatment groups to prove the safety of this combined treatment.

Due to the precision of SBRT targeting and the minimal damage to adjacent normal structures, complications are considered to be manageable within the safe dose range. SBRT complications are related to tumor location and size. For lung lesions, the common complications are: radiation pneumonitis, radiation esophagitis, tracheal fistula, bronchial stenosis, brachial plexus injury, hemoptysis, and chest pain (28). The only complication in our patient was grade I radiation pneumonitis, which was visualized by CT as a small area of pulmonary fibrosis around the irradiation focus, suggesting that SBRT safety was acceptable (Figure 1B). Further, there were several novel aspects to this case report. Firstly, the encouraging outcomes in our case are noteworthy as the response duration following SBRT exceeded that of denosumab, which is currently recognized as the most effective systemic treatment modality. Secondly, SBRT showed better intervention adherence because it had a shorter course of treatment compared to systematic treatments that require regular drug injections. Thirdly, it is the first case to discuss the possible synergistical effects of hypofractionated radiotherapy and low-dose radiation in GCTB. However, this case had several limitations, most notably the lack of biological samples from our patient before and after SBRT to explore potential changes in TME. Further, our study involved a single case, and more clinical cases need to be collected to confirm our conclusion.

\section{Conclusions}

We presented a case of unresectable GCTB treated with SBRT after the failure of separate administration of denosumab and BPs. In this case, SBRT (44 Gy/4 F) of 3 metastatic lesions was performed and all regressed significantly. One other nodule outside the PTV also shrank. After 30 months of follow-up, treatment efficacy and patient quality of life remained good, with only grade I radiation-associated pneumonia. A longer response duration was observed following SBRT relative to denosumab. These findings suggested that SBRT could be effective in managing lung metastasis of GCTB. Further, low-dose radiation-induced abscopal responses may occur during this treatment, and the immune mechanism appears to contribute to GCTB regression.

\section{Acknowledgments}

The authors thank the colleagues at the Cancer Center for their help and valuable discussions.

Funding: None.

\section{Footnote}

Reporting Checklist: The authors have completed the CARE reporting checklist. Available at https://atm.amegroups. com/article/view/10.21037/atm-21-6575/rc

Conflicts of Interest: All authors have completed the ICMJE uniform disclosure form (available at https://atm. amegroups.com/article/view/10.21037/atm-21-6575/coif). The authors have no conflicts of interest to declare.

Etbical Statement: The authors are accountable for all aspects of the work in ensuring that questions related to the accuracy or integrity of any part of the work are appropriately investigated and resolved. All procedures performed in this study were in accordance with the ethical standards of the institutional and/or national research committee(s) and with the Helsinki Declaration (as revised in 2013). Written informed consent was obtained from the patient for publication of this case report and accompanying images. A copy of the written consent is available for review by the editorial office of this journal.

Open Access Statement: This is an Open Access article distributed in accordance with the Creative Commons Attribution-NonCommercial-NoDerivs 4.0 International License (CC BY-NC-ND 4.0), which permits the noncommercial replication and distribution of the article with the strict proviso that no changes or edits are made and the original work is properly cited (including links to both the formal publication through the relevant DOI and the license). See: https://creativecommons.org/licenses/by-nc$\mathrm{nd} / 4.0 /$. 


\section{References}

1. Mendenhall WM, Zlotecki RA, Scarborough MT, et al. Giant cell tumor of bone. Am J Clin Oncol 2006;29:96-9.

2. Niu X, Zhang Q, Hao L, et al. Giant cell tumor of the extremity: retrospective analysis of 621 Chinese patients from one institution. J Bone Joint Surg Am 2012;94:461-7.

3. Yang Y, Huang Z, Niu X, et al. Clinical characteristics and risk factors analysis of lung metastasis of benign giant cell tumor of bone. J Bone Oncol 2017;7:23-8.

4. Siebenrock KA, Unni KK, Rock MG. Giant-cell tumour of bone metastasising to the lungs. A long-term follow-up. J Bone Joint Surg Br 1998;80:43-7.

5. Tsukamoto S, Mavrogenis AF, Kido A, et al. Current Concepts in the Treatment of Giant Cell Tumors of Bone. Cancers (Basel) 2021;13:3647.

6. Gong T, Luo Y, Wang Y, et al. Multiple Pulmonary Metastases of Recurrent Giant Cell Tumor of Bone with Expression of VEGFR-2 Successfully Controlled by Denosumab and Apatinib: A Case Report and Literature Review. Cancer Manag Res 2021;13:4447-54.

7. Kwan JM, Cheng R, Feldman LE. Hepatotoxicity and Recurrent NSTEMI While on Pembrolizumab for Metastatic Giant Cell Bone Tumor. Am J Med Sci 2019;357:343-7.

8. de Jonge MJ, Hamberg P, Verweij J, et al. Phase I and pharmacokinetic study of pazopanib and lapatinib combination therapy in patients with advanced solid tumors. Invest New Drugs 2013;31:751-9.

9. Chawla S, Blay JY, Rutkowski P, et al. Denosumab in patients with giant-cell tumour of bone: a multicentre, open-label, phase 2 study. Lancet Oncol 2019;20:1719-29.

10. Thomas D, Henshaw R, Skubitz K, et al. Denosumab in patients with giant-cell tumour of bone: an open-label, phase 2 study. Lancet Oncol 2010;11:275-80.

11. Yasko AW. Interferon therapy for giant cell tumor of bone. Curr Opin Orthop 2006;17:568-72.

12. Abraham AK, Ramanathan M, Weinstock-Guttman B, et al. Mechanisms of interferon-beta effects on bone homeostasis. Biochem Pharmacol 2009;77:1757-62.

13. Wei F, Liu X, Liu Z, et al. Interferon alfa-2b for recurrent and metastatic giant cell tumor of the spine: report of two cases. Spine (Phila Pa 1976) 2010;35:E1418-22.

14. Schreuder WH, Peacock ZS, Ebb D, et al. Adjuvant Antiangiogenic Treatment for Aggressive Giant Cell Lesions of the Jaw: A 20-Year Experience at Massachusetts General Hospital. J Oral Maxillofac Surg 2017;75:105-18.

15. Dubey S, Rastogi S, Sampath V, et al. Role of intravenous zoledronic acid in management of giant cell tumor of bone- A prospective, randomized, clinical, radiological and electron microscopic analysis. J Clin Orthop Trauma 2019;10:1021-6.

16. Ruka W, Rutkowski P, Morysiński T, et al. The megavoltage radiation therapy in treatment of patients with advanced or difficult giant cell tumors of bone. Int J Radiat Oncol Biol Phys 2010;78:494-8.

17. Kim IY, Jung S, Jung TY, et al. Gamma knife radiosurgery for giant cell tumor of the petrous bone. Clin Neurol Neurosurg 2012;114:185-9.

18. Kim EY, Murovic JA, Gibbs IC, et al. A giant cell tumor of the cranial base treated by stereotactic radiosurgery. J Radiosurg SBRT 2012;1:333-7.

19. Qi DW, Wang P, Ye ZM, et al. Clinical and Radiographic Results of Reconstruction with Fibular Autograft for Distal Radius Giant Cell Tumor. Orthop Surg 2016;8:196-204.

20. Ito K, Tanaka H, Furuya T, et al. First report of stereotactic body radiotherapy for large-volume spinal tumors. Int Cancer Conf J 2017;6:149-53.

21. de la Peña C, Guajardo JH, Gonzalez MF, et al. Cyberknife stereotactic radiosurgery and denosumab for giant cell tumour of the skull base: Case report. Rep Pract Oncol Radiother 2017;22:429-33.

22. Weng JC, Li D, Wang L, et al. Surgical management and long-term outcomes of intracranial giant cell tumors: a single-institution experience with a systematic review. J Neurosurg 2018;131:695-705.

23. Formenti SC, Demaria S. Combining radiotherapy and cancer immunotherapy: a paradigm shift. J Natl Cancer Inst 2013;105:256-65.

24. Huang Y, Kim BYS, Chan CK, et al. Improving immunevascular crosstalk for cancer immunotherapy. Nat Rev Immunol 2018;18:195-203.

25. Abuodeh Y, Venkat P, Kim S. Systematic review of case reports on the abscopal effect. Curr Probl Cancer 2016;40:25-37.

26. Yin L, Xue J, Li R, et al. Effect of Low-Dose Radiation Therapy on Abscopal Responses to Hypofractionated Radiation Therapy and Anti-PD1 in Mice and Patients With Non-Small Cell Lung Cancer. Int J Radiat Oncol Biol Phys 2020;108:212-24.

27. Menon H, Chen D, Ramapriyan R, et al. Influence of lowdose radiation on abscopal responses in patients receiving high-dose radiation and immunotherapy. J Immunother Cancer 2019;7:237.

28. Welsh JW, Tang C, de Groot P, et al. Phase II Trial of Ipilimumab with Stereotactic Radiation Therapy for 
Metastatic Disease: Outcomes, Toxicities, and Low-Dose Radiation-Related Abscopal Responses. Cancer Immunol Res 2019;7:1903-9.

29. Klug F, Prakash H, Huber PE, et al. Low-dose irradiation programs macrophage differentiation to an iNOS $/ M 1$ phenotype that orchestrates effective $\mathrm{T}$ cell immunotherapy. Cancer Cell 2013;24:589-602.

30. Desar IM, Braam PM, Kaal SE, et al. Abscopal effect of radiotherapy in a patient with metastatic diffuse-type giant cell tumor. Acta Oncol 2016;55:1510-2.

31. Ke J, Cheng S, Yao MY, et al. Novel Strategy of Curettage and Adjuvant Microwave Therapy for the Treatment of Giant Cell Tumor of Bone in Extremities: A Preliminary Study. Orthop Surg 2021;13:185-95.

(English Language Editor: A. Muijlwijk)

Cite this article as: Feng L, Ye T, Zhang J, Yuan S, Chen Y, Chen J. Stereotactic body radiotherapy for lung metastases in a patient with giant cell tumor of bone: a case report and literature review. Ann Transl Med 2022;10(3):156. doi: 10.21037/atm-21-6575 